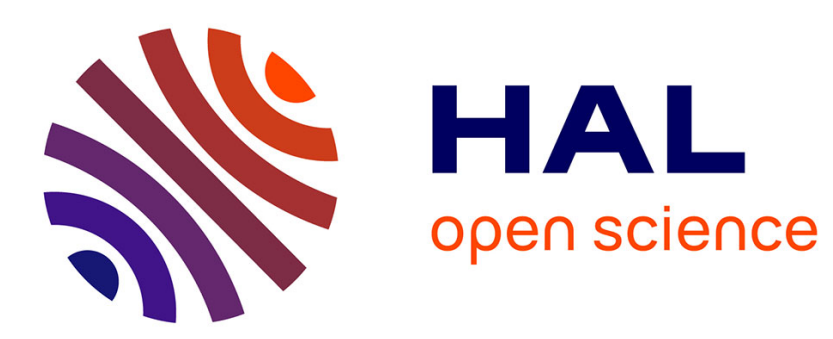

\title{
Interférométrie et réflectométrie haute résolution en lumière incohérente: modélisation d'interférogrammes
}

Cyril Lupi, Eric Tanguy, Christian Boisrobert, Frédérique de Fornel

\section{To cite this version:}

Cyril Lupi, Eric Tanguy, Christian Boisrobert, Frédérique de Fornel. Interférométrie et réflectométrie haute résolution en lumière incohérente: modélisation d'interférogrammes. Canadian Journal of Physics, 1999, pp.685-692. hal-00935132

\section{HAL Id: hal-00935132 https://hal.science/hal-00935132}

Submitted on 4 Feb 2014

HAL is a multi-disciplinary open access archive for the deposit and dissemination of scientific research documents, whether they are published or not. The documents may come from teaching and research institutions in France or abroad, or from public or private research centers.
L'archive ouverte pluridisciplinaire HAL, est destinée au dépôt et à la diffusion de documents scientifiques de niveau recherche, publiés ou non, émanant des établissements d'enseignement et de recherche français ou étrangers, des laboratoires publics ou privés. 


\title{
Interférométrie et réflectométrie haute résolution en lumière incohérente: modélisation d'interférogrammes
}

\author{
Cyril Lupi, Eric Tanguy, Christian Boisrobert et Frédérique \\ de Fornel
}

\begin{abstract}
Résumé : Nous présentons des résultats infrarouge de réflectométrie à basse cohérence dans des fibres optiques, des guides d'onde et des coupleurs afin de détecter les échos dûs aux défauts de propagation réflective et pour mesurer leurs propriétés optiques : pertes ou atténuation, dispersion. Cette technique est basée sur l'interférométrie et donne la plus haute résolution spatiale avec la plus faible puissance optique réfléchie. Nous balayons le bras de référence de notre interféromètre de Michelson autour de la position de la « frange en lumière blanche " et nous obtenons des interférogrammes dont les enveloppes et les franges contiennent l'information sur le spectre de la source de lumière et sur la réflectivité du bras test. Nous donnons des exemples et nous montrons que des simulations numériques sont nécessaires pour comprendre la signature des réflecteurs et déterminer leur structure physique.
\end{abstract}

\begin{abstract}
We report on low-coherence, near-infrared reflectometry applied to optical fibres, waveguides, and coupling devices to detect the echoes due to reflective propagation defects and measure their optical properties - losses or attenuation, dispersion. This technique, based on interferometry, leads to the highest spatial resolution and the lowest detectable reflected optical power. We scan the reference arm of our Michelson interferometer around the "white light fringe" position and obtain interferograms whose envelopes and fringes contain information on the light source spectrum and the reflectivity of the test arm. Theoretical and experimental results are compared. Examples are given and show that numerical simulations are needed to understand the signatures of the reflectors and get to their physical structures.
\end{abstract}

\section{Introduction}

La technique de mesure par réflectométrie permettant d'atteindre la meilleure résolution spatiale et la plus grande sensibilité dans les guides optiques est empruntée à de l'interférométrie en lumière blanche. Le réflectomètre utilisé est un interféromètre de Michelson réalisé à l'aide d'un coupleur à fibres, et les

C. Lupi ${ }^{1}$, E. Tanguy et C. Boisrobert. Équipe de physique des solides pour l'électronique, EA 2158, Groupe d'optique et optoélectronique, Faculté des sciences, B. P. 92208,44322 Nantes Cédex 3, France.

F. de Fornel. Laboratoire de physique de l'Université de Bourgogne, UPRES A CNRS 5027, Groupe d'optique de champ proche, Faculté des Sciences Mirande, B. P. 400, F-21011 Dijon Cédex, France.

1. Auteur correspondant : courrier électronique : Cyril.lupi@physique.univ-nantes.fr 
sources sont des diodes électroluminescentes.

Dans la première partie nous présentons d'abord les éléments essentiels de l'analyse numérique, puis nous décrivons le dispositif expérimental et évoquons les notions de temps de cohérence et de résolution spatiale.

Dans la seconde partie les résultats des calculs et les résultats expérimentaux sont comparés. Le premier exemple d'application illustre la technique de mesure de la résolution spatiale. Dans le second exemple et en reprenant l'analyse numérique proposée, nous montrons qu'il est possible de découvrir les détails essentiels des éléments de couplage à partir des interférogrammes correspondants.

Nous résumons dans la conclusion les perspectives offertes par cette approche sur le traitement de toutes les informations contenues dans les interférogrammes.

\section{Interférométrie et réflectométrie en lumière incohérente}

\subsection{Rappels théoriques}

Le champ total recueilli par le détecteur est la somme des champs réfléchis dans les bras de référence et de test. Lorsque les chemins optiques des deux bras sont égaux, l'intensité détectée est maximale. Si le miroir mobile s'écarte de la position d'équilibre de la distance $x$, il y a alors un déphasage $\phi$ entre les deux ondes :

$\phi=\frac{2 x}{c} \omega=\frac{4 \pi}{\lambda} x$

où $\omega$ est la pulsation de l'onde, $c$ la célérité de la lumière et $\lambda$ la longueur d'onde.

Pour une source monochromatique, l'intensité en fonction du déplacement du miroir mobile est une sinusoïde affectée de certains coefficients. Dans le cas plus réaliste d'une source incohérente, le champ électrique $E(t)$ satisfait l'équation des ondes, il a donc pour expression :

$E(t)=\int_{-\infty}^{+\infty} a(v) \exp (-j \omega t) \mathrm{d} \nu$

$a(v)$ étant le spectre du champ électrique.

Le spectre de puissance optique de la source est défini de la manière suivante :

$S(v)=a^{*}(v) a(v)$

En négligeant les termes de bruit, !'intensité optique détectée par la photodiode est définie comme une moyenne temporelle du module au carré du champ électrique total :

$I(x)=\left\langle\left|a_{2} E(t)+a_{3} E\left(t+\frac{2 x}{c}\right)\right|^{2}\right\rangle$

$a_{2}$ étant la proportion de l'intensité lumineuse du faisceau incident qui est couplée dans le bras test, $a_{3}$ celle couplée dans le bras de référence. Si le système est considéré sans pertes : $a_{2}^{2}+a_{3}^{2}=1$.

En développant (4) nous obtenons la forme littérale de l'interférogramme :

$I(x)=\left(a_{2}^{2}+a_{3}^{2}\right) I_{0}+2 a_{2}^{2} a_{3}^{2} \Re\left\langle E^{*}(t) E\left(t+\frac{2 x}{c}\right)\right\rangle$

$I_{0}$ est la moyenne temporelle du champ $E(t)$ multiplié par son conjugué.

L'intensité est composée d'un terme constant et d'un second qui constitue la partie détenant l'information sur l'élément réflecteur.

Par ailleurs, nous supposerons que les phases des composantes des fréquences différentes ne sont pas corrélées :

$\left\langle\mathrm{e}^{j\left(\phi(\omega)-\phi\left(\omega^{\prime}\right)\right)}\right\rangle=\delta\left(\omega-\omega^{\prime}\right)$ 
Nous aboutissons à partir de (5) et (6) à une relation directe entre l'interférogramme et le spectre de la source :

$I(x) \propto \Re\left(\int_{-\infty}^{+\infty} S(\nu) \mathrm{e}^{j \omega(2 x / c)} \mathrm{d} v\right)$

Ce qui nous permet d'affirmer que le spectre de la source est déterministe, c'est-à-dire qu'à un profil de spectre de source donné correpond un type d'interférogrammes caractéristiques de celle-ci.

Afin de mieux comprendre l'effet de la cohérence de la source sur l'interférogramme, il est nécessaire de définir le temps de cohérence de la source [1] :

$\tau_{c^{\circ}}=\int_{-\infty}^{+\infty}|\gamma(\tau)|^{2} \mathrm{~d} \tau$

$\gamma(\tau)$ étant défini par :

$\gamma(\tau)=\int_{-\infty}^{+\infty}\left(\frac{S(\nu)}{\int_{-\infty}^{+\infty} S(\nu) \mathrm{d} \nu}\right) \mathrm{e}^{2 j \pi \nu \tau} \mathrm{d} \tau$

$\tau_{C}$ représente la durée de vie moyenne des trains d'ondes émis par les sources incohérentes. Pendant cette durée, en moyenne, chaque vibration conserve son amplitude et sa phase initiale. Deux trains d'ondes issus de la même vibration et décalés dans le temps d'un retard supérieur à $\tau_{c}$ ne peuvent donner naissance à un interférogramme.

Les temps de cohérence pour différents types de spectres sont donnés par les relations suivantes [2] : spectre lorentzien :

$\tau_{c}=\frac{1}{\pi \Delta \nu}$

spectre gaussien :

$\tau_{c}=\sqrt{\frac{2 \ln 2}{\pi}} \frac{1}{\Delta v}$

spectre rectangulaire :

$\tau_{c}=\frac{1}{\Delta \nu}$

$\Delta v$ étant la largeur spectrale de la source.

Nous constatons qu'à largeur spectrale égale, la source lorentzienne offre un temps de cohérence plus court donc une meilleure résolution spatiale [5]. Afin de confirmer ces résultats, il est important de confronter les résultats numériques liés à ces expressions avec les résultats expérimentaux.

\subsection{Dispositif expérimental}

Le banc de réflectométrie utilisé est un interféromètre de Michelson [2-10] réalisé à l'aide d'un coupleur à fibres ainsi qu'il est représenté sur la figure 1 .

La source est une diode électroluminescente InGaAs/InP à spectre large, couplée à l'un des deux bras d'entrée du coupleur. Le détecteur est une photodiode PIN InGaAs/InP couplée au second bras d'entrée du coupleur. L'extrémité du bras de référence de longueur variable comporte un double système de collimation à lentilles $(\mathrm{L} 1, \mathrm{~L} 2)$. L'équipage réflecteur est constitué de la seconde lentille $\mathrm{L} 2$ et du miroir plan $M_{2}$ placé dans son plan focal. Un translateur piézoélectrique déplace ce réflecteur parallèlement à l'axe optique. $E_{2}(t)$ et $E_{3}(t)$ sont les ondes réfléchies respectivement par les bras de référence et de test (fig. 1). Elles interfèrent dans le plan de la photodiode. Retenons que la largeur à mi-hauteur de l'enveloppe de l'interférogramme est d'autant plus faible que le spectre optique de la source est plus large. La distance minimale détectable entre deux centres réflecteurs, ou résolution spatiale, est de l'ordre de $7 \mu \mathrm{m}$ lorsque la largeur spectrale de la diode électroluminescente est $50 \mathrm{~nm}$. 
Fig. 1. Montage expérimental : interféromètre tout fibre.

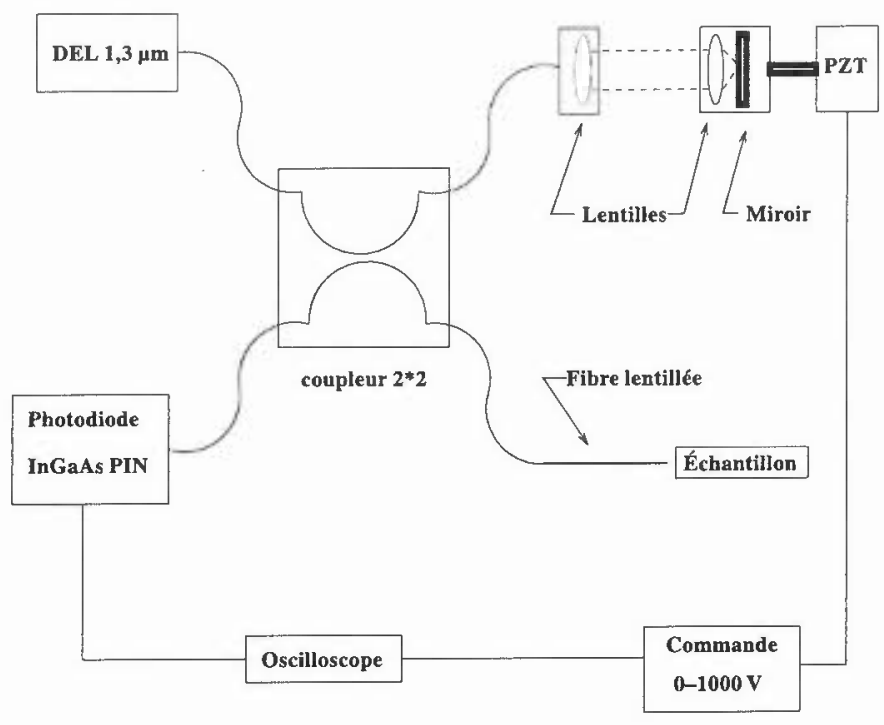

\section{Comparaison des résultats numériques et expérimentaux}

Pour une source à spectre gaussien, la réponse expérimentale de l'interféromètre donne un interférogramme à enveloppe gaussienne [7]. Le résultat en résolution spatiale, fonction de la largeur spectrale de la source abordée dans le paragraphe précédent, est retrouvé également par le calcul. Nous présentons les résultats expérimentaux (fig. 2) et théoriques (fig. 3) pour une source rectangulaire. Divers types de spectres ont été étudiés, et à chaque type de spectre de source correspond une signature particulière de l'interférogramme. La figure 3 en est un exemple caractéristique.

Les résultats ont confirmé que pour une même largeur spectrale, la meilleure résolution spatiale est obtenue à partir d'une source à spectre lorentzien.

Pour la mesure de la résolution spatiale, nous avons considéré une lame couvre-objet couplée en bout du bras test (fig. 4 et 5). L'indice de cette lame est de 1,47 et son épaisseur est de $28 \mu \mathrm{m}$. Nous avons approximé le champ réfléchi dans le bras test comme la somme de la réflexion sur la face d'entrée et celle sur la face de sortie : la longueur de cohérence de notre source étant de $18 \mu \mathrm{m}$, le champ réfléchi dans le bras test est :

$E_{2}(t)=E(t)\left[r_{1}+t_{1} r_{2} t_{-1} \mathrm{e}^{-4 j \pi\left(n_{2} d / \lambda\right)}\right]$

$r_{1}$ étant le coefficient de réflexion sur la face d'entrée, $r_{2}$ celui sur la face de sortie, $t_{1}$ le coefficient de transmission sur l'aller, $t_{-1}$ celui sur le retour, $n_{2}$ étant l'indice de la lame.

En résolvant littéralement le calcul de (5) pour une source à spectre gaussien, se propageant dans un milieu de groupe d'indice $N$ et subissant une réflexion après une distance $L$, l'intensité détectée est :

$I(x) \propto \exp \left[-\left(\frac{\Delta \nu}{2 c \sqrt{\ln 2}}(L N-x)\right)^{2}\right] \cos \left(\frac{4 \pi x}{\lambda}\right)$

La distance entre les interférogrammes correspondant à la face d'entrée et à la face de sortie représente le chemin optique parcouru, c'est-à-dire le produit $L$ par $N$ (fig. 4 et 5). 
Fig. 2. Interférogramme expérimental pour une source à spectre rectangulaire.
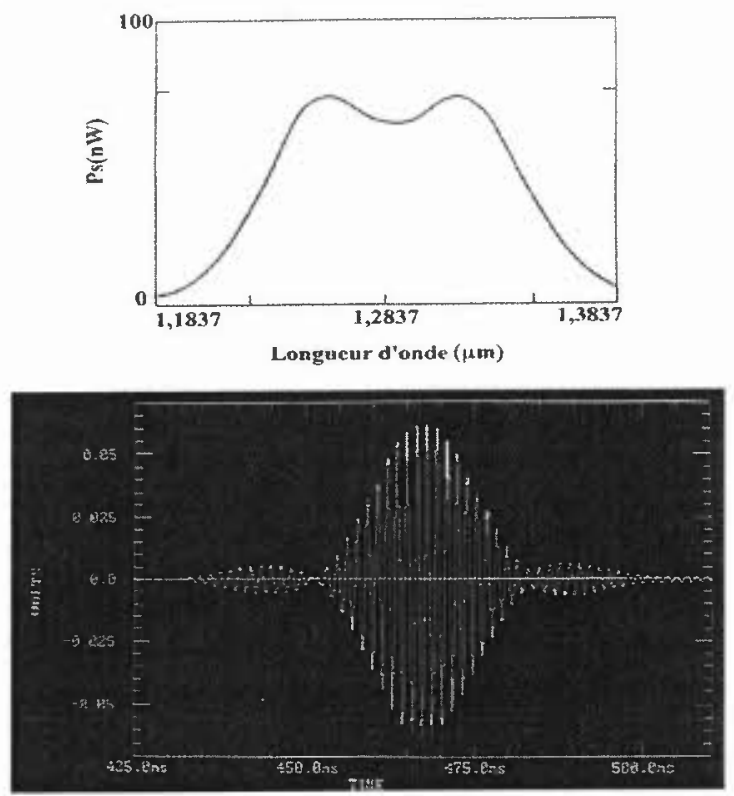

Fig. 3. Simulation numérique pour une source rectangulaire de largeur spectrale de $60 \mathrm{~nm}$.
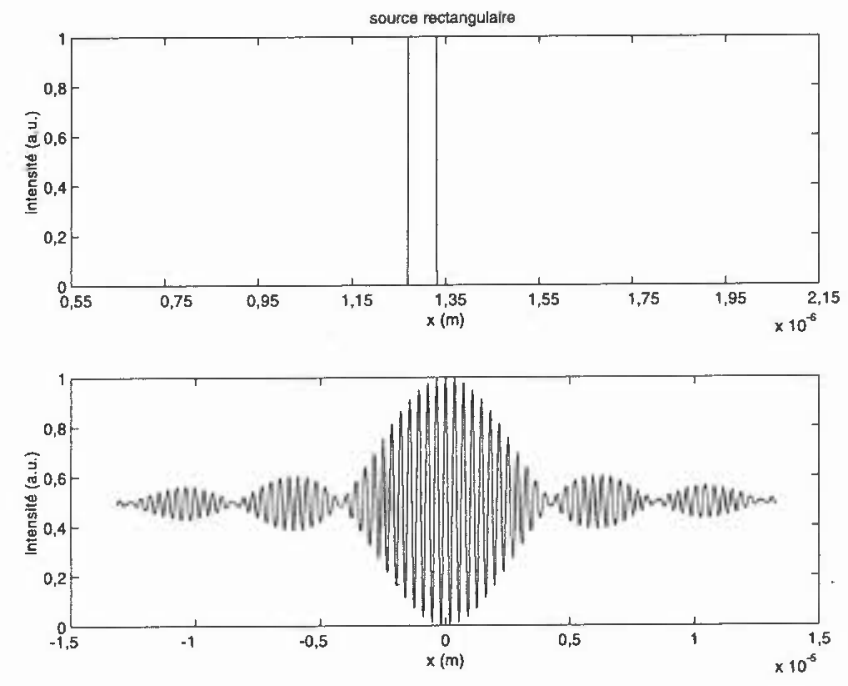

Les trains d'onde émis par la source se propagent sur une distance moyenne $L_{c}$ calculée à partir de $\tau_{c}: L_{c}=\tau_{c} c$, appelée longueur de cohérence. Ainsi pour la source utilisée la longueur de cohérence (11) est très inférieure à $L$.

Ce type de calcul permet de déterminer des signatures associées à des phénomènes de propagation 
Fig. 4. Mesure expérimentale d'une lame couvre-objet d'épaisseur de $28 \mu \mathrm{m}$ et d'indice 1,47 .

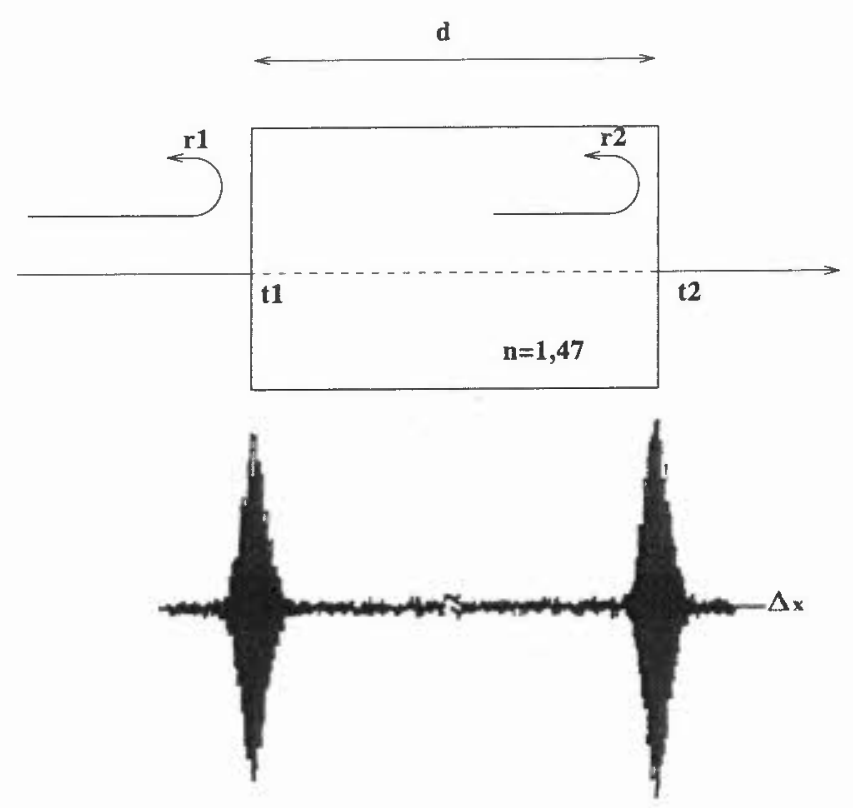

Fig. 5. Modélisation de 1' interférogramme correspondant à la lame couvre-objet.
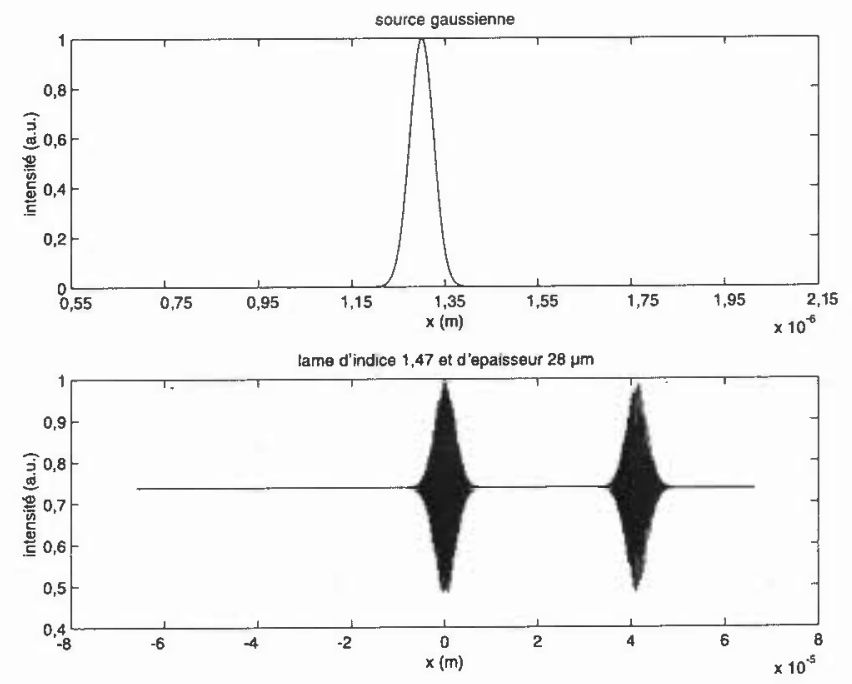

et de réflexion. Ils peuvent être complexes en raison des propriétés optiques (atténuation, réflexion, dispersion) des guides et des réflecteurs.

La figure 6 est l'illustration simple d'un dispositif de couplage laser-microlentille-fibre. Consi- 
Fig. 6. Schéma de couplage d'une puce laser à une fibre lentillée connectée au bras test de l'interféromètre.

Microlentille

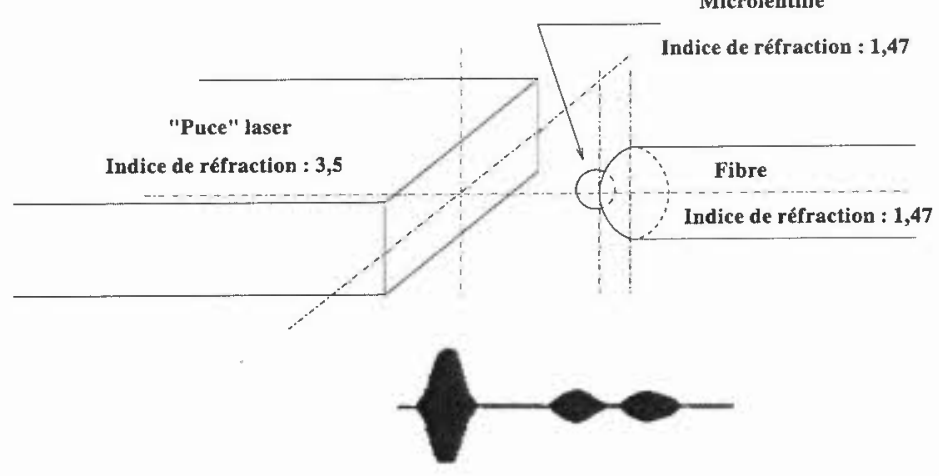

Fig. 7. Réponse expérimentale du couplage fibre lentillée - puce laser.

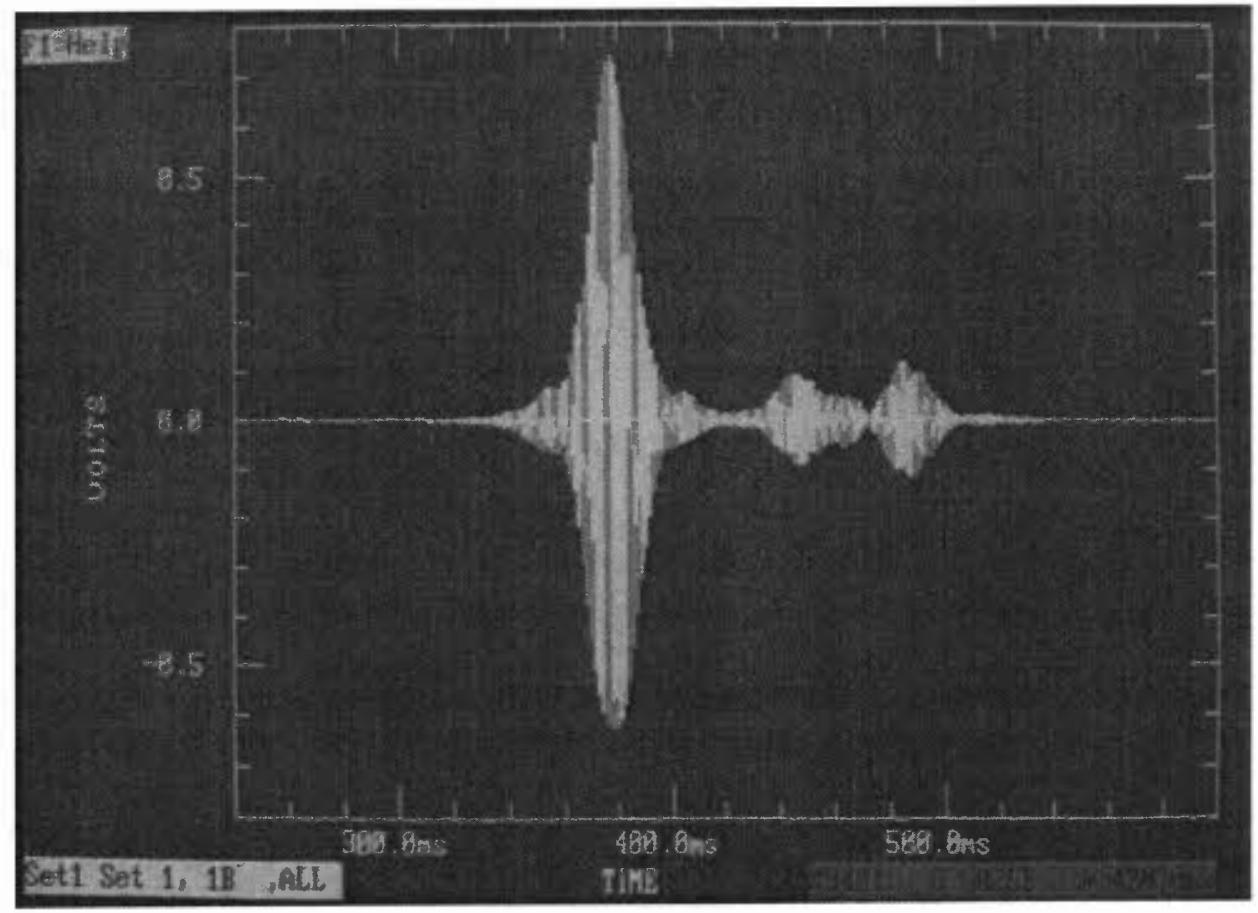

dérons l'interférogramme expérimental obtenu à l'aide d'une source à spectre rectangulaire (fig. 2) et représenté sur la figure 7. L'analyse théorique appliquée à ce dispositif permettrait de décorréler les divers phénomènes physiques contenus dans l'interférogramme. 


\section{Conclusions et perspectives}

Cette étude nous a permis de confirmer que les spectres sont déterministes. Il est fondamental de choisir la source en fonction de son spectre, de sa largeur spectrale, afin d'obtenir la résolution spatiale désirée.

Nous avons ensuite montré que chaque interférogramme relevé au cours de l'allongement du bras de référence est « la signature » d'un centre réflecteur localisé le long du bras test.

L'interférogramme entier (enveloppes et franges) contient les informations à partir desquelles nous pouvons extraire la position exacte et les propriétés optiques du centre réflecteur lorsque le spectre de la source est connu.

\section{Remerciements}

Nous tenons à remercier D. Leduc, L. Salomon et A. Rahmani pour leurs nombreux conseils. Nous sommes reconnaissants du support financier de FRANCE TELECOM et du Bureau National de Métrologie.

\section{Bibliographie}

1. L. Mandel et E. Wolf. Optical coherence and quantum optics. Cambridge University Press, 1995.

2. Allen, Politch et Young. Optical coherence domain reflectometry of integrated optical components. NIST. 1995.

3. B.L. Danielson et C.D. Whitenberg. Appl. Opt. 26, 14 (1987); 26, 2836 (1987).

4. B.L. Danielson et C.Y. Boisrobert. Appl. Opt. 30, 21 (1990).

5. E. Brinkmeyer. Opt. Lett. 17, 20 (1992); 17, 1441 (1992).

6. J.F. Lucas, L.F. DeChiaro, C. Salla et C.Y. Boisrobert. Electron. Lett. 29, (1993)

7. C.Y. Boisrobert. Thèse de l'université Montpellier II. 1994.

8. K. Takada, H. Yamada, Y. Hibino et S. Mitachi. Elect. Lett. 31, 18 (1995); 31, 1565 (1995).

9. R.J. Recknagel et G. Notni. Opt. Commun. 148, 122 (1998).

10. U. Wiedmann et P. Gallion. J. Lightwave Technol. 16, 7 (1998). 